\title{
Endovascular therapy for patients with heritable thoracic aortic disease
}

\author{
Alice Le Huu ${ }^{1}$, Jacqueline K. Olive ${ }^{1}$, Davut Cekmecelioglu ${ }^{1,2}$, Subhasis Chatterjee ${ }^{1,2}$, Hiruni S. Amarasekara ${ }^{1,3}$, \\ Susan Y. Green ${ }^{1,3}$, Joseph S. Coselli ${ }^{1,2}$, Ourania Preventza ${ }^{1,2}$ \\ ${ }^{1}$ Division of Cardiothoracic Surgery, Michael E. DeBakey Department of Surgery, Baylor College of Medicine, Houston, TX, USA; ${ }^{2}$ Section of \\ Adult Cardiac Surgery, Department of Cardiovascular Surgery, Texas Heart Institute, Houston, TX, USA; ${ }^{3}$ Office of Surgical Research, Michael E. \\ DeBakey Department of Surgery, Baylor College of Medicine, Houston, TX, USA \\ Correspondence to: Ourania Preventza, MD. Baylor College of Medicine, BCM 390, One Baylor Plaza, Houston, TX 77030, USA. \\ Email: preventz@bcm.edu.
}

Background: Patients with genetic or heritable aortic conditions and thoracic aortic aneurysm syndrome often develop cardiovascular abnormalities originating at the aortic root and affecting the entire thoracoabdominal aorta. Although thoracic endovascular aortic repair (TEVAR) is usually avoided in these patients, TEVAR may be worthwhile for those at high risk for surgical complications and in certain emergency circumstances. We explored indications for TEVAR in patients with suspected or confirmed genetic or heritable aortic conditions and investigated early and mid-term outcomes.

Methods: Our institutional aortic surgery database was queried for patients with suspected or confirmed Marfan syndrome, Loeys-Dietz syndrome, Ehlers-Danlos syndrome, Turner syndrome, neurofibromatosis, or familial aortic aneurysm and dissection who underwent TEVAR between February 1, 2002 and October 31, 2020. We extracted operative details and in-hospital, follow-up, and survival data.

Results: Thirty-seven patients who underwent 40 endovascular interventions met the inclusion criteria; 25 previously underwent ascending aorta or aortic root surgery, and 21 previously underwent open thoracoabdominal surgery. Postoperative complications included respiratory failure $(24.3 \%)$, cardiac complications $(16.2 \%)$, renal failure $(13.5 \%)$, tracheostomy $(8.1 \%)$, and spinal cord ischemia (paraplegia/ paraparesis) (8.1\%). Follow-up ranged from 1.3 to 8.5 years (median: 3.6 years), with 15 deaths overall (three early/in-hospital). Thirteen patients $(35.1 \%)$ had 22 repeat interventions (open and endovascular) postTEVAR; five had the endograft removed.

Conclusions: Despite consensus that thoracic aneurysms in patients with genetic or heritable aortic conditions should be treated with conventional open surgery, the outcomes from our study suggest that TEVAR might be suitable in emergency settings or for patients in this population who are not candidates for open surgery, who are at high risk for reintervention, or who have a previously implanted Dacron graft. Nonetheless, lifelong surveillance is important for these patients after TEVAR to monitor for new dissection at distal or proximal landing zones, as repeat interventions are frequent.

Keywords: Endovascular procedures; heritable; genetic; congenital; aneurysm; dissecting; aortic aneurysm; thoracic; genetic predisposition to disease; postoperative complications; vascular grafting

Submitted Jun 15, 2021. Accepted for publication Oct 04, 2021.

doi: 10.21037/acs-2021-taes-109

View this article at: https://dx.doi.org/10.21037/acs-2021-taes-109

(c) Annals of Cardiothoracic Surgery. All rights reserved. 


\section{Introduction}

The Genetically Triggered Thoracic Aortic Aneurysms and Cardiovascular Conditions (GenTAC) registry is a multiinstitutional, US National Institutes of Health-funded database of patients with confirmed or suspected genetically triggered thoracic aortic aneurysms or dissections. The conditions most commonly cited in the GenTAC registry include syndromic disorders such as Marfan syndrome, Loeys-Dietz syndrome, and vascular Ehlers-Danlos syndrome, familial thoracic aortic aneurysm and dissection, and early-onset dissection in patients younger than 50 years of age. Aortic valve disease has also been associated with Turner syndrome, neurofibromatosis, tuberous sclerosis, Noonan syndrome, osteogenesis imperfecta, and homocystinuria (1). Patients with these conditions are often afflicted with extensive cardiovascular abnormalities that originate at the aortic root and that can affect the entire thoracoabdominal aorta. It is not uncommon for these patients to have undergone multiple surgical interventions, either through a sternotomy to access the ascending aorta or arch or through a thoracoabdominal approach to access the descending aorta.

The use of endovascular technology to treat pathologies of the descending thoracic aorta (DTA) is well documented. In 2005, the US Food and Drug Administration approved the first stent graft to treat aneurysms confined to the DTA. Since then, various stent grafts have been approved not only for thoracic aneurysms, but also for acute and chronic dissections, coarctations, and other thoracic aortic pathologies. However, in patients with genetic or heritable aortic conditions and thoracic aortic aneurysm syndromes, the appropriate use of this emerging technology remains obscure. Concerns focus on the risk for continued radial expansion of an intrinsically vulnerable native aorta in these patients, which may increase the patient's susceptibility to retrograde dissection or new intimal tears. Additionally, conditions due to genetic factors that result in chaotic rearrangement of the extracellular matrix commonly cause multiple aortic aneurysms at risk for dissection and rupture.

Because of the complexity of the pathology and the relatively young age of the patients (often $<50$ years), these lesions can be difficult to address. Traditionally, the presence of a genetic or heritable aortic condition has been considered a contraindication to endovascular intervention. However, endovascular stent placement in a patient at prohibitive risk for surgery, when done under emergency circumstances, as a bridge to more definitive repair, or when the patient has had multiple redo procedures, may be worth considering - even in individuals with genetic or heritable aortic conditions.

We conducted a study of patients with a suspected or confirmed genetic or heritable aortic condition who had undergone thoracic endovascular aortic repair (TEVAR). Our aim was to delineate indications for use of TEVAR in these patients and to investigate early and mid-term outcomes.

\section{Methods}

\section{Patients}

In this data review study, the aortic surgery database at Baylor College of Medicine in Houston, Texas was queried for patients with Marfan syndrome, Loeys-Dietz syndrome, Ehlers-Danlos syndrome, Turner syndrome, neurofibromatosis, or familial aortic aneurysm and dissection who had undergone TEVAR between February 1, 2002 and October 31, 2020. All patients with a suspected or confirmed case of any of the above disorders were included.

In 2006, the Baylor College of Medicine Institutional Review Board (IRB) approved our prospective and retrospective collection of clinical information. Data for patients who underwent TEVAR before this IRB approval were collected retrospectively from electronic medical records. Informed consent was waived for these patients. Data for patients who underwent TEVAR after the IRB approved the research protocol were collected prospectively. Informed consent was obtained from these patients when possible; consent was waived for those with no family members or no ability to consent due to illness.

Clinical data such as detailed operative reports, admission notes, and discharge summaries were gathered and reviewed. Adverse events were documented. Review of outpatient medical records was performed to determine previous medical history, operative characteristics, followup imaging results, and subsequent interventions. To ensure that all deaths were captured, the Social Security Death Index was reviewed, and follow-up phone calls with family and primary care physicians were made.

\section{TEVAR characteristics}

The patients selected for this study had undergone a thorough preoperative review by the aortic surgery team at Baylor College of Medicine. After preoperative images were carefully reviewed, a treatment plan was formulated. Those 


\begin{tabular}{|c|c|}
\hline Variable & Values \\
\hline Median age, years & 46.5 (range, 21-72) \\
\hline Men, n (\%) & $20(54.1)$ \\
\hline Ratio of men to women & $1.2: 1.0$ \\
\hline $\begin{array}{l}\text { Previous ascending aorta or aortic root } \\
\text { surgery, } \mathrm{n}(\%)\end{array}$ & $25(67.6)$ \\
\hline $\begin{array}{l}\text { Previous descending or thoracoabdominal } \\
\text { surgery, } \mathrm{n}(\%)\end{array}$ & $21(56.8)$ \\
\hline \multicolumn{2}{|l|}{ TEVAR acuity, n (\%) } \\
\hline Emergency/urgent & $17(45.9)$ \\
\hline Elective & $20(54.1)$ \\
\hline \multicolumn{2}{|l|}{ Comorbidities, n (\%) } \\
\hline Diabetes & $1(2.7)$ \\
\hline Hypertension & $31(83.8)$ \\
\hline Hyperlipidemia & $8(21.6)$ \\
\hline Coronary artery disease & $6(16.2)$ \\
\hline $\begin{array}{l}\text { Congestive heart failure (ejection } \\
\text { fraction }<50 \%)\left(n=23^{\dagger}\right)\end{array}$ & $3(8.1)$ \\
\hline Cerebrovascular disease & 7 (18.9) \\
\hline Pulmonary disease & 8 (21.6) \\
\hline History of tobacco use & $13(35.1)$ \\
\hline $\begin{array}{l}\text { Chronic kidney disease (eGFR } \\
<60 \mathrm{~mL} / \mathrm{min} / 1.73 \mathrm{~m}^{2} \text { ) }\end{array}$ & $5(13.5)$ \\
\hline Marfan syndrome, n (\%) & $27(73.0)$ \\
\hline Loeys-Dietz syndrome, n (\%) & $6(16.2)$ \\
\hline Ehlers-Danlos syndrome, n (\%) & $0(0.0)$ \\
\hline Turner syndrome, n (\%) & $2(5.4)$ \\
\hline Neurofibromatosis, n (\%) & $1(2.7)$ \\
\hline $\begin{array}{l}\text { Familial thoracic aortic aneurysm and } \\
\text { dissection, } \mathrm{n}(\%)\end{array}$ & $1(2.7)$ \\
\hline
\end{tabular}

${ }^{\dagger}$, values are missing for a few patients. TEVAR, thoracic endovascular aortic repair; eGFR, estimated glomerular filtration rate.

who were considered to be poor surgical candidates at high risk for complications from an open operation were offered TEVAR as an alternative. In addition, in certain emergency circumstances, TEVAR was performed as a bridge to subsequent open operation and as a lifesaving measure in acute settings. All procedures were performed in a hybrid endovascular suite by an experienced team of aortic surgeons.

Before 2008, thoracic repairs were performed mainly with Gore TAG stent grafts and Gore graft excluders (W. L. Gore \& Associates, Flagstaff, AZ). After 2008, other endovascular stent grafts, such as Cook Zenith TX2 grafts (Cook Medical, Inc., Bloomington, IN) and Medtronic Talent, Captivia, and Valiant grafts (Medtronic, Minneapolis, $\mathrm{MN}$ ), also were used.

\section{Analysis and statistics}

All statistical analyses were completed using Microsoft Excel (Microsoft Corporation, Redmond, WA) and SPSS 19.0 for Windows (IBM Corporation, Armonk, NY). Descriptive data are presented as numbers and percentages or medians with ranges.

\section{Results}

During the study period, 37 patients with suspected or confirmed Marfan syndrome, Loeys-Dietz syndrome, EhlersDanlos syndrome, Turner syndrome, neurofibromatosis, or familial aortic aneurysm and dissection underwent 40 endovascular thoracic aortic interventions at our institution. Patient demographics and comorbidities are shown in Table 1.

The median patient age was 46.5 years (range, $21-$ 72 years); 20 were men, and 17 were women. Of the 37 patients overall, 25 had previously undergone ascending aorta or aortic root surgery, and 21 had previously undergone open thoracoabdominal surgery. Chronic DeBakey type I aortic dissection or chronic DeBakey type III aortic dissection was present in 22 patients, and acute Type III dissection was present in four patients. Ten patients had pseudoaneurysm formation at previous repair sites, four had descending thoracic aortic (DTA) aneurysms, three had intercostal patch aneurysms, two had aortoesophageal erosion, and one had an aortobronchial fistula. Some of the patients were treated for more than one condition.

Acute type I aortic dissection occurred in three patients. In one patient with evidence of visceral malperfusion, TEVAR performed as a first approach was followed by open proximal repair. The other two patients had previously undergone DTA replacement; in these patients, an ascending repair with debranching and endovascular exclusion of the arch was performed. Specific details regarding endograft 


\begin{tabular}{ll}
\hline \multicolumn{2}{l}{ Table 2 TEVAR operative details ( $\mathrm{n}=37 ; 40$ interventions) } \\
\hline Variable & Number (\%) \\
\hline $\begin{array}{l}\text { Landing zone } \\
\text { Zone } 2 \text { (between left common carotid and left }\end{array}$ & $6(15.0)$ \\
subclavian artery) & $16(40.0)$ \\
Zone 3 (<2 cm distal to the left subclavian) & $18(45.0)$ \\
Zone 4 (>2 cm distal to the left subclavian) & $8(20.0)$ \\
Landing zone characteristics & $4(10.0)$ \\
\hline $\begin{array}{l}\text { Proximal landing zone in native aorta } \\
\text { Distal landing zone in native aorta }\end{array}$ & $15(37.5)$ \\
Both landing zones in native aorta & $13(32.5)$ \\
\hline Both landing zones inside Dacron graft &
\end{tabular}

\begin{tabular}{ll}
\hline Table 3 Postoperative complications $(\mathrm{n}=37)$ & \\
\hline Variable & Number (\%) \\
\hline 30-day death & $2(5.4)$ \\
\hline $\begin{array}{l}\text { Respiratory failure } \\
\text { Need for tracheostomy }\end{array}$ & $9(24.3)$ \\
\hline $\begin{array}{l}\text { Renal failure requiring hemodialysis (persistent } \\
\text { and transient) }\end{array}$ & $3(8.1)$ \\
\hline $\begin{array}{l}\text { Persistent renal failure requiring hemodialysis } \\
\text { Spinal cord injury (paraplegia and paraparesis) }\end{array}$ & $2(5.4)$ \\
\hline Stroke & $3(8.1)$ \\
\hline Bleeding requiring reoperation & $0(0.0)$ \\
\hline $\begin{array}{l}\text { Cerebrovascular complications (subdural } \\
\text { hematoma) }\end{array}$ & $1(2.7)$ \\
\hline Cardiac complications & $1(2.7)$ \\
\hline
\end{tabular}

landing zones are shown in Table 2.

Postoperative complications are detailed in Table 3. There were two deaths within 30 days of surgery and one death after months of prolonged hospital course. One patient with Marfan syndrome had previously undergone open repair of a ventricular septal defect and a repair of chronic Type III dissection of the DTA. We performed a redo sternotomy to repair an aneurysm of the aortic root and ascending proximal aortic arch, during which we placed a Freestyle bioroot prosthesis (Medtronic) and replaced the ascending and the proximal arch. Due to pseudoaneurysm formation at the level of the previous DTA anastomosis, the patient also required stent placement exactly distally to the left subclavian artery. Extracorporeal membrane oxygenation (ECMO) support was needed postoperatively; however, the patient was unable to be weaned from ECMO, and the family withdrew support.

A second patient had a history of an infected DTA Dacron graft that was replaced with homografts. One of the homografts subsequently ruptured, necessitating emergency thoracotomy and repair of the aorta. The patient presented a few months later with the same symptoms and underwent endovascular repair of the ruptured homograft instead of having another thoracotomy. The patient had a prolonged postoperative course complicated by multiorgan failure and infection; as a result, the family withdrew support.

The third patient also had Marfan syndrome and had previously had a Bentall operation for acute type I aortic dissection. This patient underwent extent III thoracoabdominal aortic aneurysm repair. Due to postoperative bleeding from the anastomosis site, an endograft was placed inside the Dacron graft. The patient's postoperative course was complicated by multiorgan failure and a subdural hematoma that necessitated a craniotomy. The patient was transferred to an extendedcare rehabilitation facility and died there a few months later.

Overall, our median follow-up was 3.6 years (range, 1.3-8.5 years); 15 deaths were noted during the followup period, including one death that was related to late reintervention. Thirteen patients (35.1\%) had 22 (59.5\%) repeat interventions; eight of these patients required only one follow-up procedure after their index TEVAR, whereas five patients had multiple repairs, both endovascular and open, after their index TEVAR. Of the 22 repeat interventions, 17 were open procedures and five were endovascular procedures. Open procedures included thoracoabdominal aortic repair and descending thoracic aortic repair; the stent graft was extracted in five patients and was fully salvaged in two other cases.

\section{Discussion}

In this study, we analyzed a cohort of patients with suspected or confirmed Marfan syndrome, LoeysDietz syndrome, Ehlers-Danlos syndrome, Turner syndrome, neurofibromatosis, or familial aortic aneurysm and dissection who underwent TEVAR. We used this endovascular technology for patients who we considered to 
be at high risk for complications from an open operation, and in emergency situations as a bridge to subsequent open operation or as a lifesaving measure. The early outcomes were acceptable for this subset of patients.

On a cellular level, patients with genetic or heritable aortic condition and thoracic aortic aneurysm syndromes have a fragile aortic wall that is vulnerable to the radial stress exerted by a stent graft. This can lead to progression of the aneurysm or propagation of a dissection if the proximal or distal stent landing zone is on native aorta. Stent migration due to the abnormal aortic tissue also is of concern. Patients with genetic or heritable aortic conditions are poor candidates for demonstrating the safety of TEVAR devices, because the use of TEVAR is always off-label in these patients, given that they are usually excluded from device trials due to their young age and friable aortas.

The available data on these patients and endovascular stenting are also limited by having come from studies with small sample sizes; consequently, statistical analysis of the findings is challenging. According to the GenTAC registry, TEVAR was performed in 31 patients [13 suspected cases and 18 confirmed cases of genetic tissue disorders, such as aneurysmal degeneration of a residual dissected DTA and acute Type A (chronic DeBakey type I or II) and acute and chronic Type B (chronic DeBakey type III) aortic dissections] (2).

The few available studies regarding TEVAR in patients with genetic or heritable aortic conditions have yielded sobering results: $\mathrm{Up}$ to $25 \%$ of patients in the GenTaC registry had new dissections after stent placement (retrograde into the arch or extending to the abdominal aorta) (2), and between $18 \%$ and $44 \%$ of patients eventually required conventional surgery; even more alarming was a $25 \%$ mortality rate (3-5). The most recent data, published by Qato et al. in 2020 (4), were more encouraging: in 102 patients with genetic tissue disorder (86\% Marfan syndrome, 9\% Ehlers-Danlos syndrome, and 5\% LoeysDietz syndrome), a lower mortality rate of $5 \%$ and an endoleak rate of $27 \%$ were observed at an average 15.6-month follow-up. This high endoleak rate at followup is consistent with the rates reported by Nordon et al. and Eid-Lidt et al. (33\% and 44\%, respectively) (6,7).

These outcomes emphasize that TEVAR is not a durable solution in patients with genetic or heritable aortic conditions. As a result, the current consensus is that thoracic aneurysms in these patients should be treated with conventional open surgery (8). Although the in-hospital mortality rate with redo aortic surgery and complex aortic pathologies is high ( $4.1 \%$ to $15.4 \%$ ), it is acceptable compared with the relative unknowns of endovascular repair (9-11).

Nonetheless, the decision to use TEVAR in patients with genetic or heritable aortic conditions becomes more nuanced when the patient is not a candidate for open surgery or is at very high risk for reintervention. In addition, patients who present with life-threatening acute Type B dissections with malperfusion may be reasonable candidates for TEVAR as a bridge to a future definitive procedure. However, lifelong surveillance and follow-up is recommended for these patients after TEVAR in the event a new dissection appears at the distal or proximal landing zone. Every effort is being made to obtain yearly follow-up imaging (computed tomographic angiography or magnetic resonance angiography for radiation reduction). Reintervention after the TEVAR can be frequent in these patients (2). In our study, 13 patients (35.1\%) had repeat reinterventions, and five patients $(13.5 \%)$ had the stent removed during follow-up open intervention.

Ideally, patients with genetic or heritable aortic conditions and a previously implanted Dacron graft are better candidates for TEVAR if the proximal and distal landing zones of the endostent would lie within the existing graft. The Dacron graft may exert a protective effect by decreasing the radial stress of the endograft on the friable aorta. If one of the landing zones is inside the Dacron graft and the other landing is on native aorta, then the latter should be monitored carefully for new dissection or aneurysm formation.

\section{Conclusions}

The endovascular devices available on the market today were not designed for use in patients with a heritable or genetic aortic condition. Future research focusing on flexibility and the "stiffness" of current designs may produce stent grafts that exert less radial force and that are therefore more suitable for primary repair in these patients. Until then, the use of stent graft technology in individuals with a genetic or heritable aortic condition should be limited to patients who are at extreme high risk or who have lifethreatening conditions (as a bridge to future definitive repair) or to patients with a previously implanted Dacron graft (where the stent lies within).

\section{Acknowledgments}

Jeanie F. Woodruff, BS, ELS, of the Scientific Publications 
\& Grants Department at the Texas Heart Institute, contributed to the editing of the manuscript.

Funding: None.

\section{Footnote}

Conflicts of Interest: Dr. OP provides consultation for and participates in clinical trials with Medtronic and W. L. Gore \& Associates. Dr. SC has participated in advisory boards for Edwards Lifesciences \& La Jolla Pharmaceutical Corp. Dr. JSC participates in clinical trials with and/or consults for Terumo Aortic, Medtronic, and W. L. Gore \& Associates and receives royalties and grant support from Terumo Aortic. The other authors have no conflicts of interest to declare.

Open Access Statement: This is an Open Access article distributed in accordance with the Creative Commons Attribution-NonCommercial-NoDerivs 4.0 International License (CC BY-NC-ND 4.0), which permits the noncommercial replication and distribution of the article with the strict proviso that no changes or edits are made and the original work is properly cited (including links to both the formal publication through the relevant DOI and the license). See: https://creativecommons.org/licenses/by-nc-nd/4.0/.

\section{References}

1. Cury M, Zeidan F, Lobato AC. Aortic disease in the young: genetic aneurysm syndromes, connective tissue disorders, and familial aortic aneurysms and dissections. Int J Vasc Med 2013;2013:267215.

2. Shalhub S, Eagle KA, Asch FM, et al. Endovascular thoracic aortic repair in confirmed or suspected genetically triggered thoracic aortic dissection. J Vasc Surg
2018;68:364-71.

3. Pacini D, Parolari A, Berretta P, et al. Endovascular treatment for type B dissection in Marfan syndrome: is it worthwhile? Ann Thorac Surg 2013;95:737-49.

4. Qato K, Conway A, Lu E, et al. Outcomes of thoracic endovascular aneurysm repair (TEVAR) in patients with connective tissue disorders. Vasc Endovascular Surg 2020;54:676-80.

5. Preventza O, Mohammed S, Cheong BY, et al. Endovascular therapy in patients with genetically triggered thoracic aortic disease: applications and short- and midterm outcomes. Eur J Cardiothorac Surg 2014;46:248-53; discussion 253.

6. Nordon IM, Hinchliffe RJ, Holt PJ, et al. Endovascular management of chronic aortic dissection in patients with Marfan syndrome. J Vasc Surg 2009;50:987-91.

7. Eid-Lidt G, Gaspar J, Meléndez-Ramírez G, et al. Endovascular treatment of type $B$ dissection in patients with Marfan syndrome: mid-term outcomes and aortic remodeling. Catheter Cardiovasc Interv 2013;82:E898-905.

8. Svensson LG, Kouchoukos NT, Miller DC, et al. Expert consensus document on the treatment of descending thoracic aortic disease using endovascular stent-grafts. Ann Thorac Surg 2008;85:S1-41.

9. Dossche KM, Tan ME, Schepens MA, et al. Twenty-four year experience with reoperations after ascending aortic or aortic root replacement. Eur J Cardiothorac Surg 1999;16:607-12.

10. Estrera AL, Miller CC, 3rd, Porat E, et al. Determinants of early and late outcome for reoperations of the proximal aorta. Ann Thorac Surg 2004;78:837-44; discussion 844-45.

11. Girardi LN. Reoperative repair of the aortic root and ascending aorta. Tex Heart Inst J 2011;38:680-3.
Cite this article as: Le Huu A, Olive JK, Cekmecelioglu D, Chatterjee S, Amarasekara HS, Green SY, Coselli JS, Preventza O. Endovascular therapy for patients with heritable thoracic aortic disease. Ann Cardiothorac Surg 2022;11(1):3136. doi: 10.21037/acs-2021-taes-109 